\title{
An Expression for Nonlinear Noise in Optical Phase Conjugation Systems with Lumped Amplifiers
}

\author{
Abdallah A. I. Ali, Christian S. Costa, Mohammad A. Z. Al-Khateeb, Filipe M. Ferreira, \\ and Andrew D. Ellis, Member, IEEE.
}

\begin{abstract}
We develop an analytical formula for evaluating the impact of fiber nonlinearity in systems employing optical phase conjugation with multi-span lumped amplification, highlighting the detailed balance of effects leading to compensation when the phase conjugator is added to the middle of the link. We apply the approach to the effect of adding a pre-dispersion element to improve the symmetry, deriving the required optimum predispersion. The closed-form is validated through simulation and shows good matching with the results within a margin of error less than $0.2 \mathrm{~dB}$.
\end{abstract}

Index Terms-Four-wave mixing, optical fiber communication, optical phase conjugation, fiber nonlinear optics.

\section{INTRODUCTION}

O PTICAL phase conjugation (OPC) is an all optical signal processing technique for the mitigation of dispersive and Kerr effect based nonlinear effects in single mode fiber (SMF). By placing the OPC in the middle of a symmetric link, the signals phase is conjugated, reversing both the even ordered dispersion and Kerr-induced nonlinear effects in the second half of the link [1], and giving the possibility of compensation. Perfect compensation should be achieved if the dispersion-power profile is symmetric with respect to the OPC position, which is difficult to achieve in a lumped amplification system. There are different approaches to maximize the symmetry in mid-link OPC systems. One method is to use short span lengths which monotonically increases the symmetry as spacing decreases. However, this increases the required number of amplifiers and whilst energy consumption initially reduces [2], the optimum spacing will be a trade-off between energy consumption and symmetry. Another approach is to use distributed Raman amplification with either first [3] or second order [4] pumping, or even Raman amplification with short spacing [5]. In order to upgrade installed optical links with Erbium-doped fiber amplifiers (EDFAs), it is necessary to mitigate asymmetry. In this context, Minzioni et al. proposed [6] introduction of a dispersion element collocated with the OPC to enhance the symmetry by aligning the regions where the fiber nonlinearity has large impact to the same accumulated

This work was supported in part by the EPSRC (Grant EP/L000091/1PEACE).

Abdallah A. I. Ali, Christian S. Costa, Mohammad A. Z. AlKhateeb, Filipe M. Ferreira and Andrew D. Ellis are with Aston Institute of Photonic Technology (AIPT), Aston University, Birmingham, B4 7ET, UK (e-mail: aliaai@aston.ac.uk, c.sanchez-costa@aston.ac.uk, alkhamaz@aston.ac.uk, f.ferreira@aston.ac.uk, andrew.ellis@ aston.ac.uk ).

The data underlying this publication can be found at https://doi.org/10.17036/researchdata.aston.ac.uk.00000364

Copyright (C2018 IEEE dispersion. The design details were estimated from effective lengths but, despite efforts to model nonlinearity in OPC based systems [7]-[9], no rigorous analysis was presented. However, the nonlinearity in the conventional multi-span system is well modelled, whether using time-domain analysis where the nonlinearity impacts have been characterized as a phase noise [10] or using the frequency domain where the nonlinearities have been considered as an additive Gaussian noise [11], [12]. We have recently presented an analytical form for the residual nonlinearity in an OPC system [13] which differs from the analysis in [9] based on nonlinear transfer function of optical fiber. In this letter, we extend this work by developing an analytical form for the nonlinear noise in systems with mid-link OPC with and without pre-dispersion. The derived analytical expressions are validated through simulations and are used to predict the performance of a Nyquist super-channel wave division multiplexing (WDM) system. For the particular system studied, our results show that a pre-dispersed OPC improves the performance by a further $1.9 \mathrm{~dB}$ on top of the $0.8 \mathrm{~dB}$ improvement from OPC alone.

\section{THEORETICAL ANALYSis}

\section{A. FWM in mid-link OPC system with Pre-dispersion}

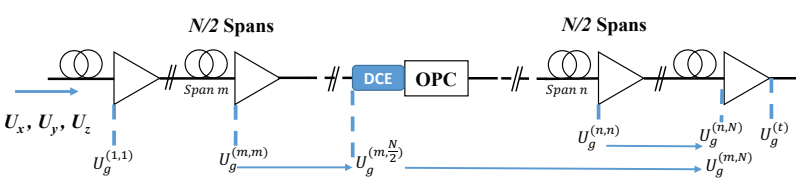

Fig. 1: Block diagram for mid-link OPC system with predispersion. DCE: Dispersion Compensating Element, $U_{g}^{(1,1)}$ : the FWM field from the first span and N: Number of spans

Figure.1 shows the modelled system, with $\mathrm{N}$ identical standard SMF (SSMF) spans of length L, dispersion $\beta_{2}$, attenuation coefficient $\alpha$ and nonlinear coefficient $\gamma$. An EDFA at the end of each span compensates the attenuation. An ideal OPC (no frequency conversion or insertion loss) is inserted in the middle of the link (based on the reported frequency-shift free [14] and low penalty OPC [4]) and a dispersion compensating element (DCE) with total dispersion $\delta$ is inserted before the OPC. For simplicity, we assume that the DCE has length $L_{d}$ and dispersion $\beta_{2 d}$ where $\delta=\beta_{2 d} L_{d}$. The dispersion element can be realized using, for example, a fiber Bragg grating based dispersion compensation device.

We assume that signal depletion is negligible, so an analytical form for the FWM power resulting from the interactions 
of three signal frequency components $U_{x}, U_{y}$ and $U_{z}$ at frequencies $f_{x}, f_{y}$ and $f_{z}$ with powers $P_{x}, P_{y}$ and $P_{z}$ can be found from the solution of the wave equation [15]. For spans before the OPC, the FWM field resulting from the propagation of the three components in span $m$ can be written as,

$$
\begin{aligned}
U_{g}^{(m, m)}= & j 2 \gamma\left(U_{x} U_{y} U_{z}^{*} e^{(m-1)\left(\frac{3}{2} \alpha+j\left(\Delta \beta+\beta_{g}\right)\right) L} G^{\frac{3}{2}(m-1)}\right) \\
& e^{\left(-\frac{\alpha}{2}+j \beta_{g}\right) L} \frac{e^{(-\alpha+j \Delta \beta) L}-1}{-\alpha+j \Delta \beta}
\end{aligned}
$$

Where $U_{g}^{(j, k)}$ is the generated FWM field from span $j$ measured at the end of span $k$ and $\beta_{g}$ is the propagation constant of the FWM field. $\Delta \beta$ is the phase mismatch and G is the EDFA field gain. $U_{g}^{(m, m)}$ will linearly propagate to the middle of the link (non-depletion approximation [15], [16]) through $\frac{N}{2}-m$ spans and then through the DCE. Following that, $U_{g}^{\left(m, \frac{N}{2}\right)}$ is conjugated and linearly propagates in the second half of the spans. Assuming that the amplifiers are exactly compensating the span loss, $U_{g}^{(m, N)}$ can be written as,

$$
\begin{aligned}
U_{g}^{(m, N)}= & -j 2 \gamma U_{x}^{*} U_{y}^{*} U_{z} e^{-j(m-1) \Delta \beta L} e^{-\frac{\alpha}{2} L} e^{-j \beta_{g d} L_{d}} \\
& \frac{e^{(-\alpha-j \Delta \beta) L}-1}{-\alpha-j \Delta \beta}
\end{aligned}
$$

Where $\beta_{g d}$ is the propagation constant of the FWM field in the DCE. Similarly, for the spans after the OPC, the FWM field generated from span $n$ can be written as,

$$
\begin{aligned}
U_{g}^{(n, N)}= & j 2 \gamma U_{x}^{*} U_{y}^{*} U_{z} e^{j(n-N-1) \Delta \beta L} e^{-\frac{\alpha}{2} L} e^{-j\left(\Delta \beta_{1}+\beta_{g d}\right) L_{d}} \\
& \frac{e^{(-\alpha+j \Delta \beta) L}-1}{-\alpha+j \Delta \beta}
\end{aligned}
$$

Where $\Delta \beta_{1}$ is the equivalent phase mismatch for the DCE. By summing the FWM fields from all the spans at the end of the link [16] and considering the last amplifier, the total FWM field from all the spans $U_{g}^{(t)}$ can be written as,

$$
\begin{gathered}
U_{g}^{(t)}=\left(\sum_{m=1}^{\frac{N}{2}} U_{g}^{(m, N)}+\sum_{n=\frac{N}{2}+1}^{N} U_{g}^{(n, N)}\right) G \\
U_{g}^{(t)}=-4 \gamma U_{x}^{*} U_{y}^{*} U_{z} e^{-j N \frac{\Delta \beta L}{4}} e^{-j \frac{\Delta \beta_{1} L_{d}}{2}} e^{-j \beta_{g d} L_{d}} \\
\frac{1}{\left(\alpha^{2}+\Delta \beta^{2}\right)} \frac{\sin \left(\frac{N \Delta \beta L}{4}\right)}{\sin \left(\frac{\Delta \beta L}{2}\right)} \kappa \\
\kappa=\alpha\left(e^{-\alpha L} \sin \left(\frac{\Delta \beta L-\Delta \beta_{1} L_{1}}{2}\right)+\sin \left(\frac{\Delta \beta L+\Delta \beta_{1} L_{1}}{2}\right)\right) \\
+\Delta \beta\left(e^{-\alpha L} \cos \left(\frac{\Delta \beta L-\Delta \beta_{1} L_{1}}{2}\right)-\cos \left(\frac{\Delta \beta L+\Delta \beta_{1} L_{1}}{2}\right)\right)
\end{gathered}
$$

From which the corresponding nonlinear FWM product power $\left(P_{g}^{(t)}=\left|U_{g}^{(t)}\right|^{2}\right)$ can be found [13].

\section{B. Non-linear Noise Power Spectral Density}

In order to calculate the total nonlinear noise power at a given frequency, we integrate $P_{g}^{(t)}$ over the signals total bandwidth, subject to the constraint that $f_{g}$ (the idler frequency) matches the frequency of interest [12], reducing the triple integral to a constrained double integral. It may clearly be integrated numerically, although this becomes impractical for large bandwidths, and gives little insight into the maximum possible nonlinearity compensation. Assuming that we have a continuous spectrum, such as Nyquist WDM channels, we slice the bandwidth to small bandwidth spectrum components with equal frequency separation $\Delta f$, then we can calculate the nonlinear noise power spectral density (PSD) $I_{\zeta}$ that affects frequency $x$, by finding the summation of the FWM PSD $I_{g}^{(t)}$ $\left(I_{g}=\frac{P_{g}}{\Delta f}\right)$ generated from the nonlinear interaction between the signal at frequency $x$ with all the other frequency components of the spectrally sliced WDM system [12]. Following a similar approach in [12] for converting the summation to integration and considering that $I_{g}^{(t)}$ is an even function (with respect to frequency), the integration can be written as,

$$
I_{\zeta}=2 \int_{0}^{\frac{B}{2}} \int_{0}^{\frac{B}{2}}\left|U_{g}^{(t)}\left(f_{1}, f_{2}\right)\right|^{2} d f_{1} d f_{2}
$$

and $\Delta \beta$ in Eq.(5) becomes $\Delta \beta=4 \pi^{2} \beta_{2} f_{1} f_{2}$. For large bandwidth, the $I_{g}^{(t)}$ is small and can be neglected because of $\Delta \beta^{4}$ in the denominator of $I_{g}^{(t)}$ and the large phase mismatch, therefore the integration boundaries for $d f_{1}$ can be changed to $0 \rightarrow \infty$.

$$
I_{1}=\int_{0}^{\infty} \frac{1}{\left(\alpha^{2}+\Delta \beta^{2}\right)^{2}} \frac{\sin ^{2}\left(\frac{N \Delta \beta L}{4}\right)}{\sin ^{2}(\Delta \beta L)} \kappa^{2} d f_{1}
$$

In order to solve the integration for $d f_{1}$ in Eq.(8), $\kappa^{2}$ in Eq.(6) needs to be expanded, the phase array term expressed as a series of cosines [12], and substitute $f=f_{1} f_{2}$ and the integration is evaluated using Cauchy's formula using the following form,

$$
\frac{1}{n !} \oint \frac{g(f)}{(f-a)^{n+1}} d f=j 2 \pi g^{(n)}(a)
$$

Where $g^{(n)}(a)$ is the $\mathrm{n}^{\text {th }}$ derivative of $g(f)$ evaluated at $a$ and $g(f)$ is analytical function over the upper half of the complex plane, $a$ is constant. The second integration is calculated as follows,

$$
I_{\zeta}=\frac{\gamma^{2} N}{\pi\left|\beta_{2}\right|} \zeta I^{3} \int_{0}^{\frac{B}{2}} \frac{1}{f_{2}} d f_{2}
$$

Where $I$ is the launch power density (assuming $P_{x}=P_{y}=$ $P_{z}=P$ ). Since the log function is not defined at 0 , we assume that the integration boundaries start from very small value $B_{\epsilon}$ compared with the signal bandwidth [12]. We arrive at the following closed expressions for the nonlinear noise PSD:

$$
I_{\zeta}=\frac{\gamma^{2} N \ln \left(\frac{B}{B_{\epsilon}}\right)}{\pi\left|\beta_{2}\right|} \zeta I^{3}
$$

Where $\zeta$ can be written as,

$$
\zeta=\zeta_{\frac{N}{2}}-\zeta_{O P C}
$$


Where $\zeta_{\frac{N}{2}}$ scales the nonlinear noise from the half of the spans and can be written as,

$$
\zeta_{\frac{N}{2}}=\frac{1-e^{-2 \alpha L}}{2 \alpha}
$$

Eq.(11) shows that the OPC insertion affects only $\zeta$ while the logarithmic dependence of the bandwidth still the same. It is worth mentioning that the more complex form, $h_{e}$ in Eq. (22) in [12] will reduce to $\left(1-e^{-2 L}\right)$ in case the integration is solved without making the assumption of large span length. $\zeta_{O P C}$ represents the OPC nonlinearity compensation with the pre-dispersion and can be written as,

$$
\zeta_{O P C}=L e^{-\left(1-\beta_{x}\right) \alpha L}\left(\beta_{x} e^{-\alpha L}\left(1-\frac{2}{N}\right)-\beta_{x}+1\right)
$$

Where $\beta_{x}$ represents the ratio of the pre-dispersion to single span accumulated dispersion and is usually less than one and can be written as,

$$
\beta_{x}=\frac{\left|\beta_{2 d}\right| L_{d}}{\left|\beta_{2}\right| L}
$$

Excess dispersion $\left(\beta_{x}>1\right)$ would tend to decorrelate the conjugate signal from the signal before the OPC and rapidly degrade the performance. Setting $\beta_{x}=0$, gives the compensation due to the OPC only. The optimum pre-dispersion that gives the minimum nonlinear noise $I_{\zeta}$ can be obtained by finding the value of $\beta_{x}$ that minimize the value of $\zeta$. Using Eq.(12) and solving the equation $\frac{\partial \zeta}{\partial \beta_{x}}=0$, the condition for optimum pre-dispersion can be written as,

$$
\beta_{x}=\frac{1}{1-e^{-\alpha L}\left(1-\frac{2}{N}\right)}-\frac{1}{\alpha L}
$$

For large span length $\left(e^{-\alpha L} \approx 0\right), \beta_{x}$ can be reduced to $\left(1-\frac{1}{\alpha L}\right)$ or $\left|\beta_{2 d}\right| L_{d} \approx\left(L-L_{e f f}\right)\left|\beta_{2}\right|$, (where $L_{e f f}$ is the effective length of SSMF) which proves the results concluded from graphical analysis in [6].

\section{Simulation Results AND Discussion}

In order to validate the analytical results, we simulated a Nyquist WDM system using Matlab for the signal generation, recovery and performance measurement and VPITransmissionMaker 9.8 for fiber transmission and optical components. The simulated system employed 17 channels of 28-Gbaud PM-16QAM (on $30 \mathrm{GHz}$ grid) which were transmitted over 10x100 km SSMF with dispersion, attenuation and nonlinear coefficients of $16 \mathrm{ps} / \mathrm{nm} / \mathrm{km}, 0.2 \mathrm{~dB} / \mathrm{km}$ and 1.3 (w.km) $)^{-1}$, respectively. An ideal OPC (no insertion loss or frequency conversion) was implemented in Matlab and inserted in the middle of the link. The transmitted bits were $2^{19}$ bits per polarization, and the simulations were conducted with 64 samples per symbol. The polarization mode dispersion (PMD) and dispersion slope were omitted because they are outside the scope of this study. An EDFA (with 5dB noise figure) was used at the end of each span to compensate the fiber loss.

Root-raised-cosine filters with a roll-off factor of 0.05 were used for pulse shaping. A preamble (1024 bits) containing constant amplitude zero autocorrelation sequences for time synchronization and channel estimation purposes was transmitted together with the information data. At the receiver, time synchronization was performed using the Schmidl \& Cox autocorrelation metric, while fine-time synchronization and channel impulse response estimation were performed using cross correlation with the training sequence. The signal was pre-dispersed before the OPC and the insertion loss for the predispersion element was ignored. The residual dispersion due to the pre-dispersion was compensated by the digital signal processing at the receiver. The performance was compared using the signal to noise ratio (SNR), either calculated for the theoretical results from the equation,

$$
\begin{gathered}
S N R=\frac{I}{I_{A S E}+I_{\zeta}} \\
I_{A S E}=0.5 F\left(e^{\alpha L}-1\right) h \nu
\end{gathered}
$$

where $I_{A S E}$ represents the amplified spontaneous emission noise PSD, F is the noise figure, $h$ is Planck constant and $\nu$ is the light frequency, or extracted from direct error counting according to (for PM-16QAM signal),

$$
B E R=\frac{3}{8} \operatorname{erfc}\left(\sqrt{\frac{S N R}{10}}\right)
$$

Three systems were investigated: without OPC (red squares), with OPC in the middle of the link (purple diamonds), and with OPC and pre-dispersion (green circles) before the OPC with the optimum accumulated dispersion (1256 ps/nm) calculated from Eq.(16). Theoretical results using Eq.(11) and Eq.(17) are plotted as lines for the three different cases. When no OPC is used, $\zeta$ is replaced by $\zeta_{\frac{N}{2}}$ in Eq.(11) with $\mathrm{N}=2 \mathrm{~N}$.

Figure (2) shows the obtained SNR as a function of the total launch power density (measured at the input of the fiber).

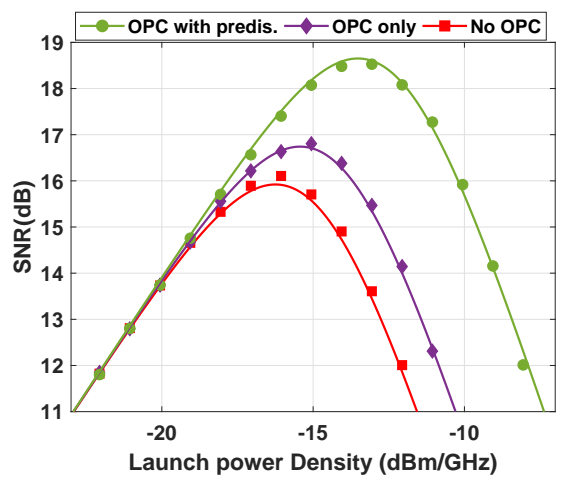

Fig. 2: The signal to noise ratio (SNR) of the central channel of 17 WDM channels as a function of the total launched power density in three cases: Without OPC (red squares), with OPC only (purple diamonds) and with OPC and pre-dispersion (green circles) for theory (lines) and simulation (markers).

We can observe that the simulation results match with the theory for the three cases. The difference between the simulated results and the analytical results is smaller than $0.12 \mathrm{~dB}$ at the optimal launch power density. For this system, the improvement due to OPC alone is less than $0.8 \mathrm{~dB}$, but increases to $2.7 \mathrm{~dB}$ due to the improved symmetry when pre-dispersion is employed. 
Next, we simulated different pre-dispersion values to verify the optimum pre-dispersion. Figure (3) shows the maximum SNR for the central channel as a function of the predispersion which closely matches the predictions of Eq.(16). The results show also that even adding a pre-dispersion less than the optimum value still improves the system performance.

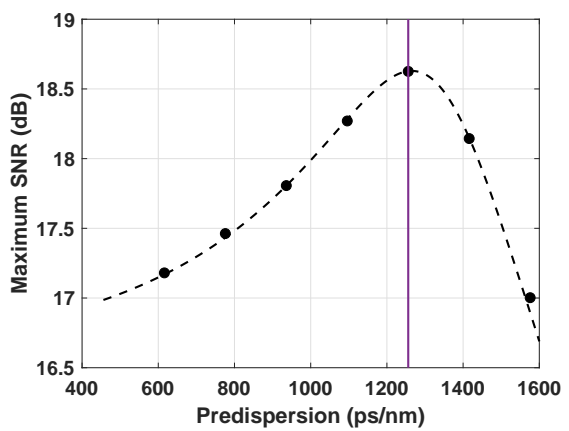

Fig. 3: The Maximum signal to noise ratio (SNR) of the central channel of 17 WDM channels as a function of the pre-dispersion for theory (dashed line) and simulation (markers). The optimum predispersion (vertical line).

Finally, we simulated the system over different span lengths for a 10 span system (variable transmission reach) for the three cases (without OPC (red), with OPC only (purple) and with OPC accompanied with pre-dispersion (green)). The SNR was estimated from the error vector magnitude (EVM) because the received signal was error-free in the high SNR region. For each simulation point, a power sweep was performed with a step size of $0.5 \mathrm{~dB}$, enabling the optimum to be estimated. For the theoretical results, the optimum launch power, and maximum SNR was calculated by differentiating Eq.(17) with respect to launch power density.

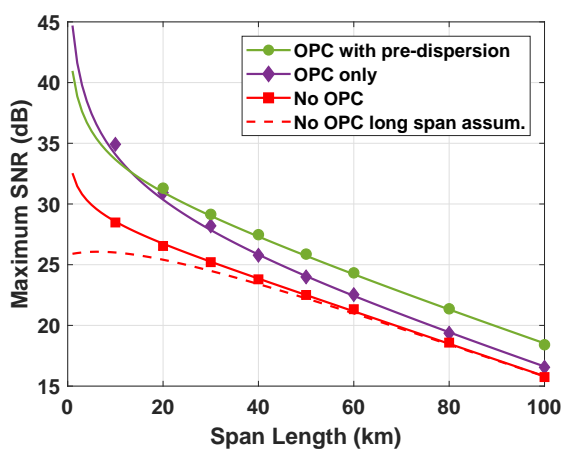

Fig. 4: The Maximum signal to noise ratio (SNR) of the central channel of 17 WDM channels as a function of the span length in three cases: Without OPC (red), with OPC only (purple) and with OPC and pre-dispersion (green) for theory (lines) (Eq.(11) (solid), Eq.(21) in [12] (dashed)) and simulation (markers).

The simulation results match the analytical predictions with a difference of less than $0.2 \mathrm{~dB}$. In addition, whilst the benefit of OPC becomes increasingly significant for short span lengths because the symmetry condition has been improved, the beneficial effect of pre-dispersion starts to decrease for spans shorter than $50 \mathrm{~km}$ and the pre-dispersion is not effective for spans less than the effective length of the SSMF and this is the reason for the cross-over in Fig.(4). The dotted line shows the analytical predictions for systems without OPC under the approximation of long span lengths, which clearly breaks down, as expected, below 40km.

Although we have assumed OPC with zero penalty in deriving Eq.(11), any additional penalty from the OPC can be added to the denominator of Eq.(17). In addition, by comparing the numerical integration of Eq.(5) with the results from Eq.(11), the model works for bandwidth greater than $250 \mathrm{GHz}$ with less than $0.3 \mathrm{~dB}$ difference from the numerical integration, and the difference starts to increase with decreasing the bandwidth as the large bandwidth assumption has been violated. For channels with large guard bands, these guard bands might need to be considered into the integration boundaries.

\section{CONCLUSiOnS}

We have introduced an analytical model to evaluate the nonlinear noise in a system employing a mid-link OPC. The model can be used to predict the amount of improvement due to the insertion of OPC and also the impact of adding a dispersion element with the OPC to improve the symmetry. The results have been validated through simulation and show good agreement.

\section{REFERENCES}

[1] A. Yariv et al., "Compensation for channel dispersion by nonlinear optical phase conjugation," Opt. Lett., vol. 4, pp. 52-54, 1979.

[2] N. Doran and A. Ellis, "Optical link design for minimum power consumption and maximum capacity," in Proc. ECOC. London, 2013.

[3] S. Jansen et al., "Long-haul DWDM transmission systems employing optical phase conjugation," IEEE J. Sel. Topics Quantum Electron., vol. 12 , pp. 505-520, 2006

[4] A. D. Ellis et al., " $4 \mathrm{~Tb} / \mathrm{s}$ transmission reach enhancement using $10 \times$ $400 \mathrm{~Gb} / \mathrm{s}$ super-channels and polarization insensitive dual band optical phase conjugation," J. Lightw. Technol., vol. 34, pp. 1717-1723, 2016.

[5] K. Solis-Trapala et al., "Nearly-ideal optical phase conjugation based nonlinear compensation system," in Proc. OFC, W3F. 8, 2014.

[6] P. Minzioni and A. Schiffini, "Unifying theory of compensation techniques for intrachannel nonlinear effects," Opt. Express, vol. 13, pp. 8460-8468, 2005.

[7] V. Pechenkin and I. J. Fair, "Analysis of four-wave mixing suppression in fiber-optic OFDM transmission systems with an optical phase conjugation module," J. Opt. Commun. Netw., vol. 2, pp. 701-710, 2010.

[8] X. Xiao et al., "Analytical design of SPM-limited systems with optical phase conjugation," IEEE Photon. Technol. Lett., vol. 20, pp. 472-474, 2008.

[9] I. Kim et al., "Analysis of nonlinearity mitigation using spectral inversion for superchannel transmission," IEEE Photon. J., vol. 8, 2016.

[10] A. Mecozzi et al., "Analysis of intrachannel nonlinear effects in highly dispersed optical pulse transmission," IEEE Photon. Technol. Lett., vol. 12 , pp. $392-394,2000$.

[11] P. Poggiolini, "The GN model of non-linear propagation in uncompensated coherent optical systems," J. Lightw. Technol., vol. 30, pp. 3857$3879,2012$.

[12] X. Chen and W. Shieh, "Closed-form expressions for nonlinear transmission performance of densely spaced coherent optical OFDM systems," Optics Express, vol. 18, no. 18, pp. 19039-19054, Aug. 2010.

[13] A. A. I. Ali et al., "Four-wave mixing in optical phase conjugation system with pre-dispersion," in Proc. OECC \& PGC, July 2017.

[14] I. Sackey et al., "Novel wavelength-shift-free optical phase conjugator used for fiber nonlinearity mitigation in 200-Gb/s PDM-16QAM transmission," in Proc. OFC. Optical Society of America, 2017, pp. Th3J-1.

[15] K. Hill et al., "cw three-wave mixing in single-mode optical fibers," $J$. Appl. Phys., vol. 49, pp. 5098-5106, 1978.

[16] D. Cleland et al., "Precise modelling of four wave mixing products over $400 \mathrm{~km}$ of step-index fibre," Electron. Lett., vol. 28, pp. 1171-1173, 1992. 